\title{
Cloud-based people counter
}

\author{
Abd Kadir Mahamad', Sharifah Saon², Hamimi Hashim³, Mohd Anuaruddin Ahmadon", \\ Shingo Yamaguchi ${ }^{5}$ \\ ${ }^{1,2,3}$ Faculty of Electrical and Electronic Engineering, Universiti Tun Hussein Onn Malaysia, Malaysia \\ ${ }^{1,2}$ Internet of Things Focus Group, Faculty of Electrical and Electronic Engineering, \\ Universiti Tun Hussein Onn Malaysia \\ ${ }^{4,5}$ Graduate School of Science and Technology for Innovation, Yamaguchi University, Japan
}

\begin{tabular}{|c|c|}
\hline Article Info & ABSTRACT \\
\hline Article history: & \multirow{10}{*}{$\begin{array}{l}\text { Emergence of Industry } 4.0 \text { in current economic trend promotes the usage } \\
\text { of Internet of Things (IoT) in product development. Counting people } \\
\text { on streets or at entrances of places is indeed beneficial for security, tracking } \\
\text { and marketing purposes. The usage of cameras or closed-circuit television } \\
\text { (CCTV) for surveillance purposes has emerged the need of tools for } \\
\text { the digital imagery content analysis to improve the system. The purpose } \\
\text { of this project is to design a cloud-based people counter using Raspberry Pi } \\
\text { embedded system and send the received data to ThingSpeak, IoT platform. } \\
\text { The initial stage of the project is simulation and coding development using } \\
\text { OpenCV and Python. For the hardware development, a Pi camera is used } \\
\text { to capture the video footage and monitor the people movement. Raspberry Pi } \\
\text { acts as the microcontroller for the system and process the video to perform } \\
\text { people counting. Experiment have been conducted to measure } \\
\text { the performance of the system in the actual environment, people counting } \\
\text { on saved video footage and visualized the data on ThingSpeak platform. }\end{array}$} \\
\hline Received Aug 30, 2019 & \\
\hline Revised Oct 28, 2019 & \\
\hline Accepted Dec 3, 2019 & \\
\hline Keywords: & \\
\hline CCTV & \\
\hline Cowd management & \\
\hline Internet of Things (IoT) & \\
\hline People counting & \\
\hline Raspberry Pi & \\
\hline
\end{tabular}

This is an open access article under the CC BY-SA license.

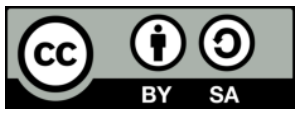

\section{Corresponding Author:}

Abd Kadir Mahamad,

Faculty of Electrical and Electornic Engineering,

Universiti Tun Hussein Onn Malaysia,

86400, Parit Raja, Batu Pahat, Johor, Malaysia.

Email: kadir@uthm.edu.my

\section{INTRODUCTION}

A crowd is defined as a number of people gathered together in a disorderly or unsystematic way. There is necessity to observe, track and perceive the behavioural of people especially in public places such as malls and stadium where the safety of the surrounding and security purposes are the main concerns. With such large number of people, it is impossible for the human surveillance operator to precisely observe the behaviour of the crowd. Hence, crowd management system that are able to perform people count ing are being designed for the purpose of facilitating the human surveillance operator in crowd management.

The usage of cameras and digitizers for different purposes has been blooming over the past few years. One of the purposes includes the use of camera or closed-circuit television (CCTV) for surveillance purposes, beside that, [1] shown that the CCTV can be used for the traffic management and routing solution of the smart parking system. This has emerged the need of tools for the digital imagery content analysis. Counting people on streets or at entrances of places is indeed beneficial for security, tracking and marketing purposes. Earlier surveillance systems using CCTV require human operator to monitor the camera's input. It has become a necessity to develop an automatic people counter to aid into the action. 
In this project, real-time people counter system using Pi camera and Raspberry Pi is proposed. Thus, the camera is set up to be hung from the ceiling to get an overhead footage of people entering or leaving the area of research. Algorithm of counting the passing people is developed and being described. At the end of this project, the captured data are shared and analysed on the cloud platform .

\section{A REVIEW OF PEOPLE COUNTER}

People counter is in high demand as it is used mainly for safety purposes, security and business. Some of people counting application are crowd management during Hajj in Makkah [2] and number of customers for business purpose. There are various methods in counting people. The methods can be classified into three approaches na mely contact-type counters, sensors implemented system, and vision-based system using a camera [3]. Contact-type counters or mechanical counters which need human contact such as turnstiles and mat-type foot switches that can obstruct the path [4]. However, this type of counter only applicable for minimal people counting and it is not suitable for massive number of people, otherwise, may cause congestion in a high-density traffic. There are numbers of sensors used in people counter done by previous projects and researches such as Pyroelectric Infrared (PIR) sensors [5-8], ultrasonic sound distance sensor [9] and thermal sensor [10]. PIR sensors counts people when they pass through the area of observation (i.e. gate and door) which is mounted with a pair of IR transceiver. The sensing device form a straight line which may cause counting error if any placing deviation occur. A previous research [9] uses ultrasonic sound distance sensor to improve accuracy and avoiding counting error. The sensor only being mounted on one side of the door which provide less possibility of displacement problem. Besides that, system uses thermalsensor only able to observe the crowd density without counting them accurately [10].

Earlier people counting mechanism which uses vision-based system suffers one problem as they unable to count the passing people precisely except when there is only a person passing through the gate at one time [11]. One of the proposed method uses stereo camera $[12,13]$ to get the vision. Stereo camera system able to count height and performing real-time 3D reconstruction [12]. Furthermore, the person and the road region on images obtained are able to be segmented accurately [13] but it removes the uses of only a single standard camera. Multiple camera is used in the system proposed in [14, 15] to solve the problem of undercounting the people walking together. Problem arises as the multiple cameras system, might have counted the same person several times as they move around the area of observation [14]. Method proposed in [15] uses approach to count crowds but they do not being individually tracked.

Crowd monitoring develop management strategies to insure public safety and evade crowd related incident. At the same time, real-time crowd monitoring helps in creating an area that deals with crowds in a simple way efficiently. Furthermore, there are other application benefited from real-time monitoring such as real-time system for monitoring activity among the elderly [16] and real-time monitoring of indoor temperature distribution for advanced air-conditioning control [17]. Earlier visual surveillance system simply uses CCTV provide visual footage and information to a human operator. People detection algorithms ca $\mathrm{n}$ be categories into two main categories which rely on whether they work in real time or not [18]. The two systems are clearly differentiated even the same approach is used in each case. Focusing on the topic, real-time monitoring system usually get initial candidate's location using image segmentation. There are several approaches; some uses human detection and tracking with acclusion [19], while the other method uses stereo vision or 3D information [20]. However, due to computational limitation, these approaches usually employ simplified person models such as ellipse and human shape templates. Others possible solution of crowd management implementation are, the mobile phone system with Bluetooth readings analysis to estimate crowd density [21] and mobile application with RFID technology [2].

Besides that, a realistic crowd scenario for aid of crowd management can be done by simulate the data captured using computer vision techniques; that can be divided into three categories which are pixel-based, texture analysis, and object-level analysis [22]. Pixel-based methods depend on very local features such as individual pixel, that generally focused on the data's density estimation, but not able to count people accurately. An earlier work of pixel-based computer vision technique to obtain data from crowd automatically is described in [23]. However, the system is not effective if high occlusions happen. People counting algorithms which implement feature extraction and pattern predicting techniques is better compared to pixel-based methods as this method requires the image patches analysis [22]. However, the method does not identify a person individually but rather focuses on counting people in a scene. A previous research proposed the crowd counting algorithm which rely on texture-based motion segmentation technique and Gaussian process regression [24]. The crowd is initially segmented into various motion directions using the mixture of dynamic textures. Then, the internal edge features, segment features and texture features are extracted from each motion frame. The method produces good output but not working properly for random crowds with wrong motion as it depends on the segmentation step. 
While, object-level analysis worked by identifying individual objects in the area. Compared to previously mentioned algorithm analysis method, this method tends to give a more accurate result. A pedestrian detection scheme using a top-down segmentation approach is proposed in [25]. Algorithm proposed able to spot and localize pedestrians in crowded area and with severe overlaps. However, in very crowded area, the lateral camera setup used can creates numbers of occlusions. A lot of methods and algorithm have been used, regarding people counting system with different techniques. Thus, this research proposed cloud-based people counter using Raspberry Pi embedded system and send the received data through Internet of Things (IoT) platform.

\section{RESEARCH METHOD}

Figure 1 shows the flowchart of proposed method. Development of this real-time people counter was divided into three phases which are; people counting algorithm development, integration of developed algorithm into Raspberry $\mathrm{Pi}$, and the integration of gained data on ThingSpeak.

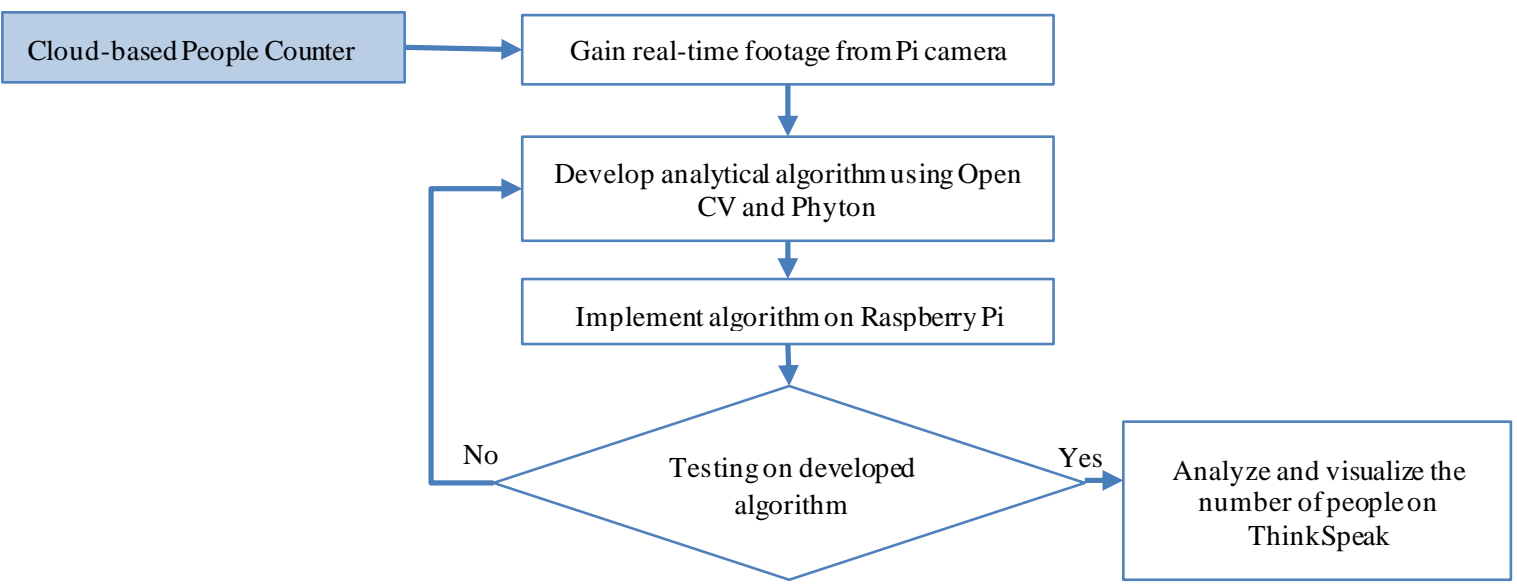

Figure 1. Flowchart of proposed method

\subsection{Phase I: development of people counting algorithm}

An analytical algorithm has been developed by using OpenCV and Python to monitor and analyse the people and their movement. There are two version of Python, namely Python 2 and Python 3. In this project, Python 3 was used to develop algorithm. There were numbers of dependencies and libraries such as CMake and Numpy installed to ensure functionality of the system. To accomplish the algorithm development, the process includes the subtraction of consecutive frames and background. After the appropriate pre-processing stage, detected objects were being tracked based on their shape, size and behaviour to assess number of people present in the video frame. These algorithms can be used to track object as building blocks or an individual object which may be helpful in this project.

\subsection{Phase II: integration of developed algorithm into Raspberry $\mathbf{P i}$}

To develop a people counter system, the algorithm developed will be integrated with the Raspberry Pi. The people-counting algorithm was deployed on Raspberry Pi for analysis of the video frame which footage was gained from the Pi camera. Pi camera was connected through Wi-Fi connection. Figure 2 shows the block diagram showing the process of coding integration into Raspberry Pi. There are several models of Raspberry Pi. The Raspberry Pi used in this project was Raspberry Pi 3 model B. This model is powered by $5.1 \mathrm{~V} / 2.5 \mathrm{~A}$ and has a quad core $1.2 \mathrm{GHz}$ Broadcom BCM2837 64bit CPU. It has a faster processor compared to previous version such as Raspberry Pi 2. Besides that, this model came with Ethernet, BCM43438 wireless LAN and Bluetooth Low Energy (BLE) on board. In this project, Wi-Fi availability was important as the system can be accessed remotely for convenience in configuration process. The operating system that was installed into Raspberry Pi is Raspbian Stretch. Raspbian Stretch has been pre-installed with numbers of software which can be used for programming, education and other general use. It has Python, Scratch, Sonic Pi, Java and more. 


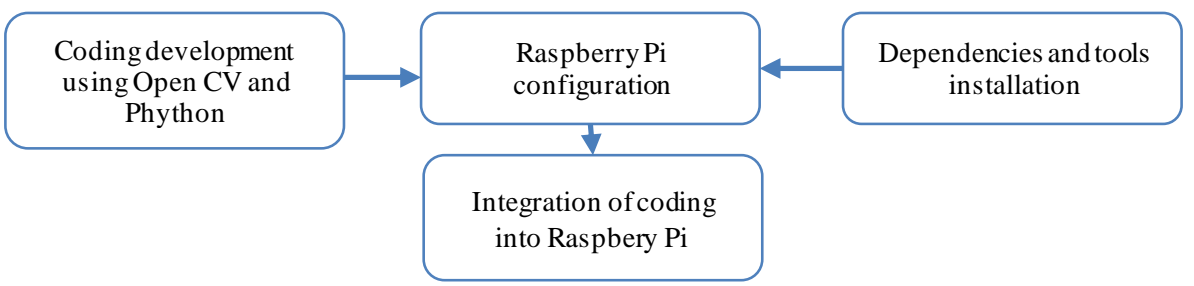

Figure 2. Block diagram of coding integration into Raspberry Pi

\subsection{Phase III: integration of data gained on ThingSpeak}

The developed algorithm then been tested on its functionality. When the algorithm was successfully developed, the data has been shared on the cloud medium, ThingSpeak. A ThingSpeak channel was created to make the data able to be shared and accessed by others. Lastly, the system performance was evaluated on its functionality whether the result obtained was as expected.

\section{RESULTS AND ANALYSIS}

Algorithm has been developed to count people by using OpenCV and Python on Raspberry Pi. In addition of the real-time Pi camera footage, a sample video was also used to test the program developed. The program was able to count the number of people that walked pass the area of research.

\subsection{Hardware development}

This device consist of main component, which are Raspberry Pi and Pi camera, as shown as in Figure 3. The device housing was made up of medium-density fiber board or better known as MDF. MDF is a lightweight material made up of dense wood fibers. The chosen material was important to ensure the device can be hang overhead on the ceiling without falling due to its weight. To keep the housing more water resistance, black-coloured spray paint was sprayed. The housing had a dimension of $11.2 \mathrm{~mm} \times 76 \mathrm{~mm} \times 50 \mathrm{~mm}$.

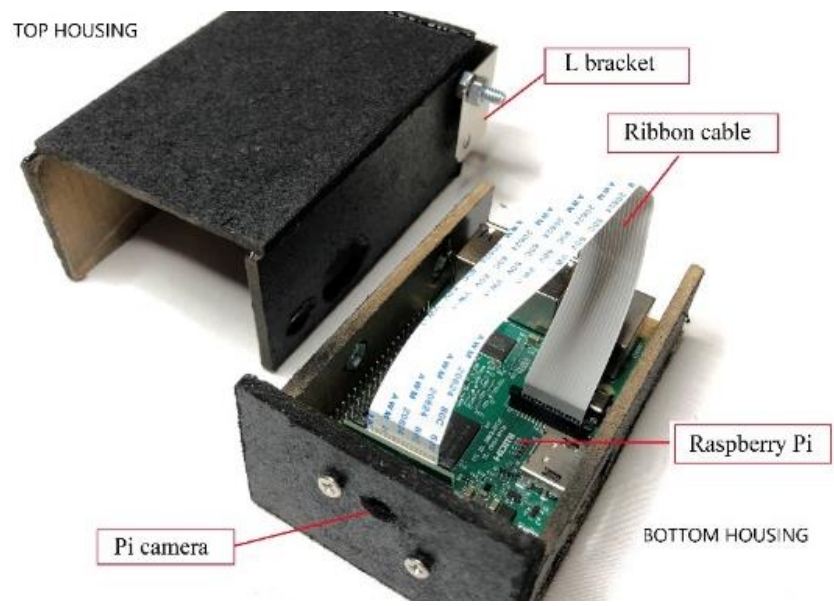

Figure 3. The cloud-based people counting device

\subsection{Algorithm development}

Python programming was used to program the Raspberry Pi. By using Windows, the code programming for Python can be done using the IDLE, the Python's Integrated Development and Learning Environment. In this project, the input for the Python came from the Pi camera and the output was the video footage gained that need to be processed and a nalysed for counting people. Figure 4 shows how the algorithm for people counting was developed.

The video input was gained in real-time on Pi camera. In addition, some alteration in algorithm also allowed saved video to be processed and analysed. The grey background was removed to differentiate the people from other visual. The frame captured was resized, converted into grayscale and the frame was 
being blurred. The detected objects can be tracked based on their shape or size to count the number of people present in the video frame. The process of detecting a person on the frame was done by contouring technique. The setup threshold is for defining a minimum area depending on the video stream. For instance, if a low threshold is set, it will form green box around the person instead of tracing the shape of people. The line code was used to form two straight lines which were a blue line for upwards direction and red line for down wards direction, as shown in Figure 5. The algorithm for the lines can be altered to make a different line colour and direction. The lines formed were used as the parameter for the counter to count the people if they walked pass it.

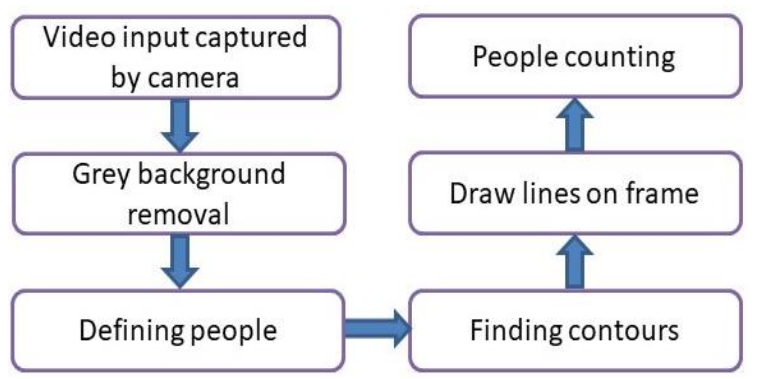

Figure 4. Algorithm development process

\subsection{System performance}

Algorithm has been developed to count people by using OpenCV and Python on Raspberry Pi. In addition of the real-time Pi camera footage, a sample video was also used to test the program developed. The program was able to count the number of people that walked pass the area of research. This outdoor experiment was carried out in surrounding with different light intensity to test on the ability of system to detect and count people. Figure 5(a) shows the initial frame at the beginning of experiment in a higher light intensity. The green box was formed as the system detected the person walking in the area of experiment. If the person walked pass the blue line (upwards direction), the count for "IN" will increase to 1. In contrary, if person walked pass red line (downwards direction), the count for "OUT" will increase to 1. The initial count for both directions are set to 0 . Figure 5(b) shows the frame captured which happen after the person walked through the lines. The counter for "IN" has increased to 1 after the person walked past the blue line. This shows that the device was able to process that someone was walking under the camera and able to count as the person walked through the line.

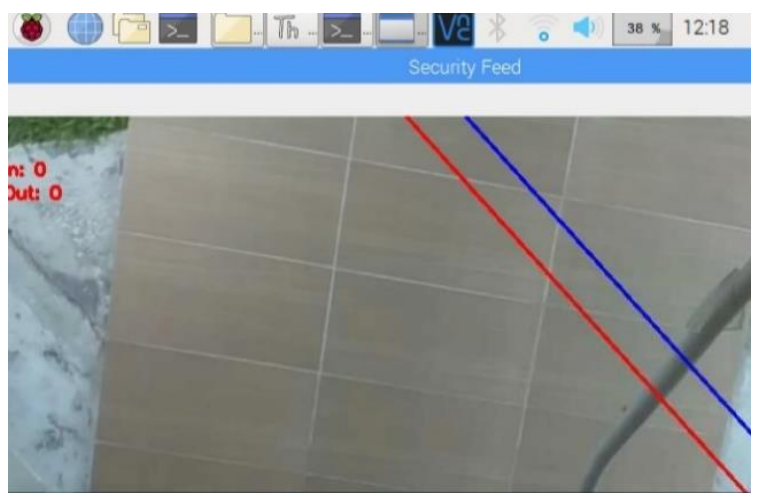

(a)

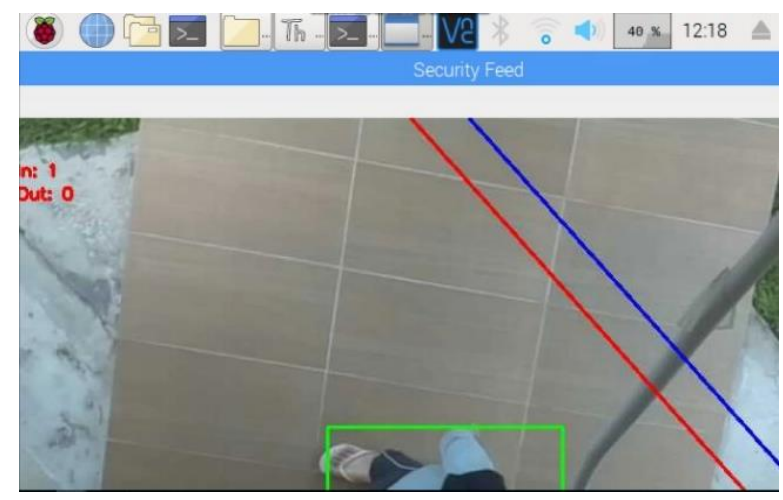

(b)

Figure 5. The camera frame, (a) At the beginning of experiment, (b) After people walked past the lines

Figure 6 shows the experiment result by using a saved video footage. In this experiment, when people walked through the blue line and walking downwards direction, the counter at top left for "DOWN" will increase subsequently. Meanwhile, if they were going upwards and walked pass the red line, the counter for "UP" will increase. Table 1 show the number of people counted during program simulation. 
However, there were several errors that occurred. This might be due to several factors that affecting the process of counting. One of the factors was the brightness of footage captured. The algorithm might not be able to do background subtraction accurately which result in object being miscounted. For example, from the video captured at 14:58:16 pm, the actual number of people walked past the line was two people, but it was only being counted as one.

The data gained was then been analysed and visualised on the cloud platform, which is ThingSpeak. Figure 7(a) and (b) show the graph of data analysed on ThingSpeak for both result for the number of people "DOWN" and "UP", respectively. For this data analysis, the data was taken from the experiment by using video footage as shown on Table 1. Data was tabulated and uploaded manually on ThingSpeak website using a CSV file. The graphs also showed the exact timestamp of when the number of people were counted.

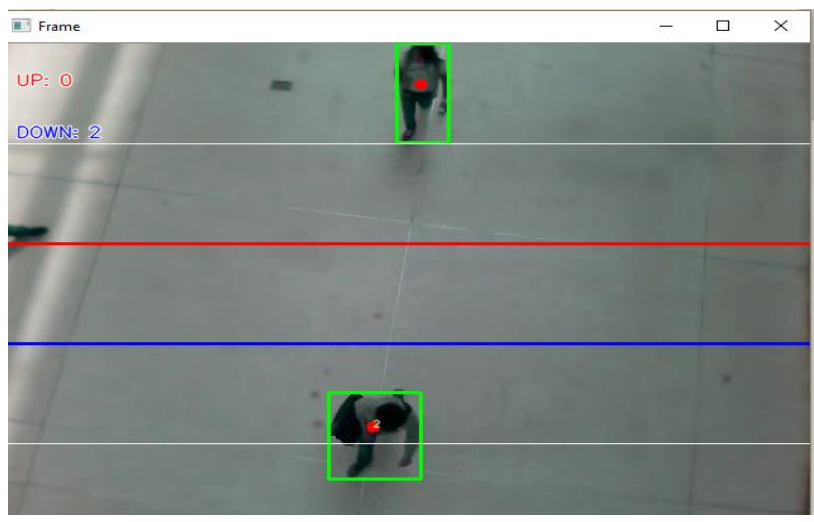

Table 1. The number of people counted during simulation

\begin{tabular}{|c|c|c|c|c|}
\hline \multirow[t]{2}{*}{$\begin{array}{l}\text { Timestamp } \\
(\mathrm{pm})\end{array}$} & \multicolumn{2}{|c|}{$\begin{array}{l}\text { Actual number of } \\
\text { people counted on } \\
\text { video footage }\end{array}$} & \multicolumn{2}{|c|}{$\begin{array}{l}\text { Total number } \\
\text { of people } \\
\text { counted from } \\
\text { simulation }\end{array}$} \\
\hline & Simulation & Actual & Down & Up \\
\hline $14: 58: 04$ & +1 & +1 & 1 & 0 \\
\hline $14: 58: 10$ & +1 & +1 & 1 & 0 \\
\hline $14: 58: 15$ & +1 & +1 & 1 & 0 \\
\hline $14: 58: 16$ & +1 & +2 & 0 & 1 \\
\hline $14: 58: 18$ & +1 & 0 & 1 & 0 \\
\hline $14: 58: 19$ & +1 & +1 & 1 & 0 \\
\hline $14: 58: 20$ & +2 & +2 & 0 & 2 \\
\hline $14: 58: 25$ & +1 & +2 & 1 & 0 \\
\hline
\end{tabular}

Figure 6. The result of video processing on a saved video footage

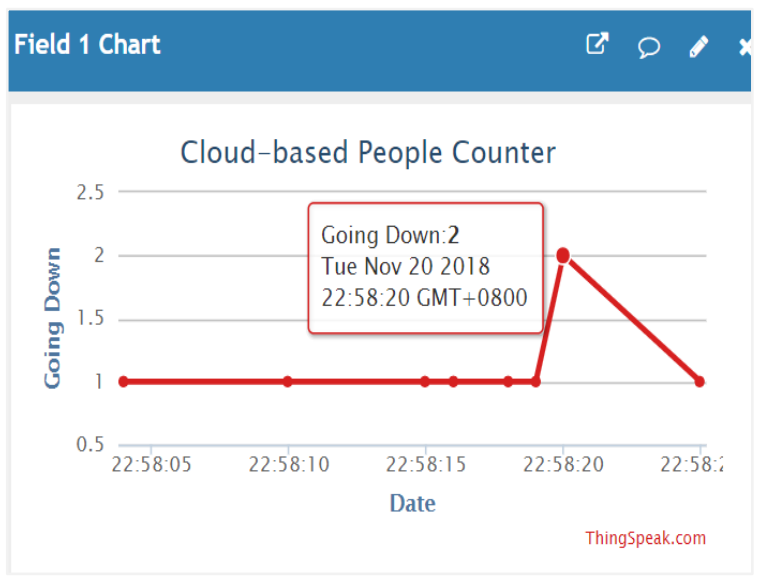

(a)

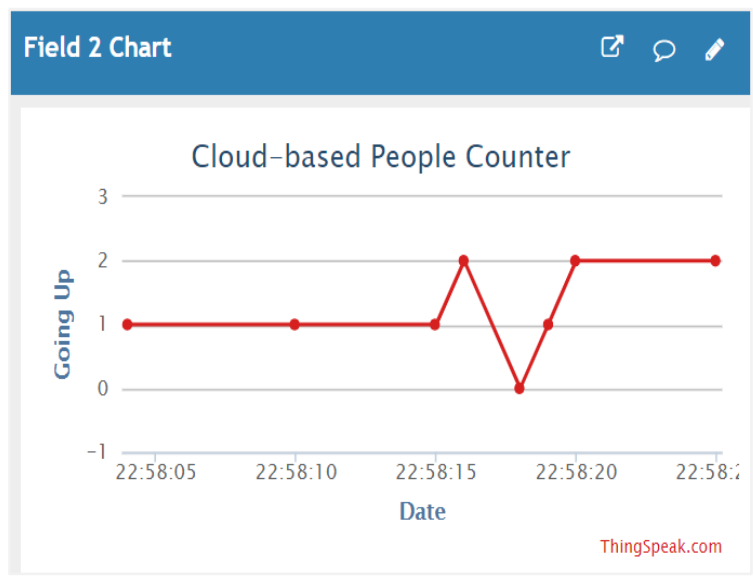

(b)

Figure 7. The graph of, (a) Number of people going downwards, (b) Number of people going upwards

\section{CONCLUSION}

Discussion in previous chapters described how the people counting system was being developed. The development method was based on analytical algorithm by using OpenCV and Python, Pi camera and a Raspberry Pi 3 with Raspbian operating system installed. The important features of this system include the ability to count people in real-time in the video footage captured and sending the statistic and data to cloud medium, ThingSpeak. The algorithm which was able to process the video received has been successfully developed by using OpenCV and Python. The developed algorithm was integrated into Raspberry Pi and the data are uploaded on ThingSpeak platform. However, there are some unfortunate limitation that caused the outcome to not fulfil some of the objectives for this experiment. 


\section{ACKNOWLEDGEMENTS}

The financial support received from the TIER 1 RESEARCH GRANT: Vot U855, Office for Research, Innovation, Commercialization and Consultancy Management (ORICC), Universiti Tun Hussein Onn Malaysia is gratefully acknowledged.

\section{REFERENCES}

[1] Z. Dzulkurnain, et al., "Internet of Things (IoT) Based Traffic Management \& Routing Solution For Parking Space," Indonesian Journal of Electrical Engineering and Computer Science, vol. 15, no. 1, pp. 336-345, 2019.

[2] R. O. Mitchell, H. Rashid, F. Dawood and A. AlKhalidi, "Hajj crowd management and navigation system: People tracking and location based services via integrated mobile and RFID systems," 2013 International Conference on Computer Applications Technology (ICCAT), Sousse, pp. 1-7, 2013.

[3] S. Velipasalar, Y. Tian and A. Hampapur, "Automatic Counting of Interacting People by using a Single Uncalibrated Camera," 2006 IEEE International Conference on Multimedia and Expo, Toronto, Ont., 2006, pp. $1265-1268$

[4] V. Prabakaran, et al., "Crowd Safety: A Real Time System for Counting People," International Journal of Innovative Technology \& Creative Engineering, vol. 1, no. 1, pp. 6-11, 2011.

[5] J. S. Yun and S. S. Lee, "Human Movement Detection and Identification Using Pyroelectric Infrared Sensors," Sensors, vol. 14, no. 5, pp. 8057-8081, 2014.

[6] F. Erden, et al., "A Robust System for Counting People Using an Infrared Sensor and A Camera," Infrared Physics \& Technology, vol. 72, pp. 127-134, 2015.

[7] Y. H. Zhang, et al., "Design of Counting System Based on Pyroelectric Infrared Sensor," Proceedings of the 2011 International Conference on Electrical, Information Engineering and Mechatronics (EIEM 2011), pp. 1343-1348, 2012.

[8] J. Yi, H. Liao, et al., "Pyroelectric Infrared Sensor and its application in People Counting System," Infrared and Laser Engineering, vol. 44, no. 1-3, pp. 1186-1192, 2015.

[9] M. S. Hossain and H. Nahiyan, "Automatic Control System for Lighting of a Single Door Room with Bidirectional People Counter," International Conference on Mechanical, Industrial and Energy Engineering, pp. 26-27, December 2014.

[10] Abuarafah, et al., "Real-time Crowd Monitoring using Infrared Thermal Video Sequences," in Journal of American Science, vol. 8, no. 3, pp. 133-140, 2012.

[11] T. Chen, T. Chen and Z. Chen, "An Intelligent People-Flow Counting Method for Passing Through a Gate," 2006 IEEE Conference on Robotics, Automation and Mechatronics, Bangkok, 2006, pp. 1-6.

[12] D. Beymer, "Person Counting using Stereo," Proc. of Workshop on Human Motion, pp. 127-133, 2000.

[13] K. Terada, D. Yoshida, S. Oe and J. Yamaguchi, "A method of counting the passing people by using the stereo images," Proceedings 1999 International Conference on Image Processing (Cat. 99CH36348), Kobe, vol. 2, pp. 338-342, 1999.

[14] V. Kettnaker and R. Zabih, "Counting people from multiple cameras," Proceedings IEEE International Conference on Multimedia Computing and Systems, Florence, Italy, vol. 2, pp. 267-271, 1999.

[15] Yang, Gonzalez-Banos and Guibas, "Counting people in crowds with a real-time network of simple image sensors," Proceedings Ninth IEEE International Conference on Computer Vision, Nice, France, vol. 1, pp. 122-129, 2003.

[16] W. Sriborrirux, P. Leamsumran and P. Dan-Klang, "Real-time system for monitoring activity among the elderly using an RF SoC device with triaxial accelerometer data over a wireless sensor network," 2014 IEEE MTT-S International Microwave Workshop Series on RF and Wireless Technologies for Biomedical and Healthcare Applications (IMWS-Bio2014), London, pp. 1-4, 2014.

[17] K. Katabira, H. Zhao, Y. Nakagawa and R. Shibasaki, "Real-Time Monitoring of People Flows and Indoor Temperature Distribution for Advanced Air-Conditioning Control," 2008 11th International IEEE Conference on Intelligent Transportation Systems, Beijing, pp. 664-668, 2008.

[18] Á. García-Martín and J. M. Martínez, "People detection in surveillance: classification and evaluation," in IET Computer Vision, vol. 9, no. 5, pp. 779-788, Oct 2015.

[19] J. Zhou and J. Hoang, "Human Detection and Tracking System," Proc. of CVPR, page 149, 2005.

[20] D. M. Gavrila and S. Munder, "Multi-cue pedestrian detection and tracking from a moving vehicle," International Journal of Computer Vision, vol. 73, no. 1, pp. 41-59, 2007.

[21] Y. Yuan, "Crowd Monitoring Using Mobile Phones," 2014 Sixth International Conference on Intelligent Human-Machine Systems and Cybernetics, Hangzhou, pp. 261-264, 2014.

[22] J. C. Silveira Jacques Junior, S. R. Musse and C. R. Jung, "Crowd Analysis Using Computer Vision Techniques," in IEEE Signal Processing Magazine, vol. 27, no. 5, pp. 66-77, Sept. 2010.

[23] A. C. Davies, Jia Hong Yin and S. A. Velastin, "Crowd monitoring using image processing," in Electronics \& Communication Engineering Journal, vol. 7, no. 1, pp. 37-47, Feb. 1995.

[24] A. B. Chan, Zhang-Sheng John Liang and N. Vasconcelos, "Privacy preserving crowd monitoring: Counting people without people models or tracking," 2008 IEEE Conference on Computer Vision and Pattern Recognition, Anchorage, AK, pp. 1-7, 2008.

[25] B. Leibe, E. Seemann and B. Schiele, "Pedestrian detection in crowded scenes," 2005 IEEE Computer Society Conference on Computer Vision and Pattern Recognition (CVPR'05), San Diego, CA, USA, vol. 1, pp. 878-885, 2005. 


\section{BIOGRAPHIES OF AUTHORS}

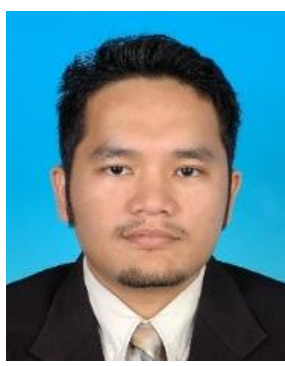

Abd Kadir Mahamad received his Bachelor of Science in Electrical Engineering (2002) and Master of Electrical Engineering (2005) from University Tun Hussein Onn Malaysia before pursuing Doctor of Philosophy (Computer Science and Electrical Engineering) at Kumamoto University, Japan (2010). He currently an Associate Professor at Faculty of Electrical and Electronic Engineering UTHM and registered as Professional Engineer. During the period of May 2015 through May 2016, he was doing industrial attachment at Melaka ICT Holdings Sdn Bhd, as Executive Assistant Manager and was involved in Smart City project in Melaka. He currently leads a research team in Video Analytic and Internet of Things (IoT). His research interests include Deep Learning, Smart City, Intelligent System applications and embedded system. He is also a Senior Member of IEEE, Institute of Engineering Malaysia (IEM) and Board of Engineering Malaysia (BEM)

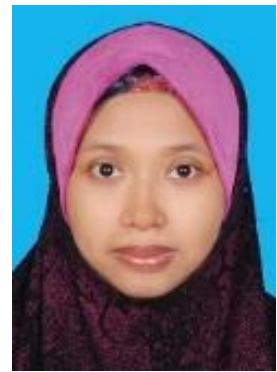

Sharifah Saon is currently a Senior Lecturer in the Faculty of Electrical and Electronic Engineering, Universiti Tun Hussein Onn Malaysia, Malaysia and registered Professional Technologists. She received the Bachelor of Science in Electrical Engineering and Master of Electrical Engineering from Universiti Teknologi Malaysia, and Kolej Universiti Tun Hussein Onn Malaysia, Malaysia, in 2001, and 2004, respectively. Her research interest is in the area of theoretical digital signal processing, visible light communication and digital \& data communication. Including the application to Internet of Things (IoT) and bigdata analysis. She is a member of IEEE, Institute of Engineering Malaysia (IEM), Board of Engineering Malaysia (BEM) and Malaysia Board of Technologists (MBOT).

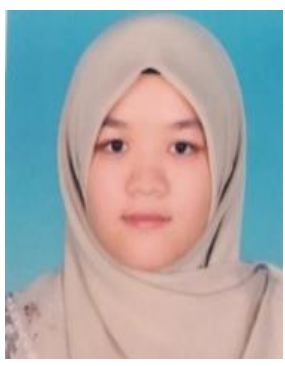

Hamimi Hashim was born on 6th of February in Wales, United Kingdom. She graduated high school from SMK Seri Tanjung, Melaka in 2012 and later furthers her studies at Johore Matriculation College. In 2014, she pursues her studies in Bachelor Degree level at Universiti Tun Hussein Onn Malaysia, Johor by majoring in Electronics Engineering.

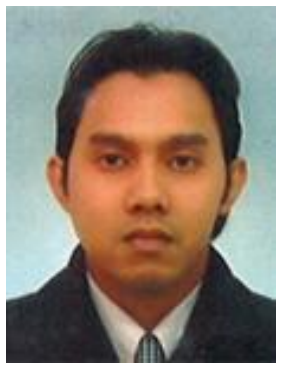

Mohd Anuaruddin Ahmadon graduated from Kumamoto National College of Technology, Japan, in 2012. He received his B.Eng. (2014), M.Eng. (2015) and Dr.Eng. (2017) from Yamaguchi University, Japan. He is currently an Assistant Professor at Graduate School of Sciences and Technology for Innovation, Yamaguchi University. He was awarded IEEE Consumer Electronics Society East-Japan Young Scientist Paper Award in 2016. His research interest includes software engineering, service engineering and its application to Internet of Things, multi-agent system and cyber-security. He is a member of IEEE and IEICE, Japan.

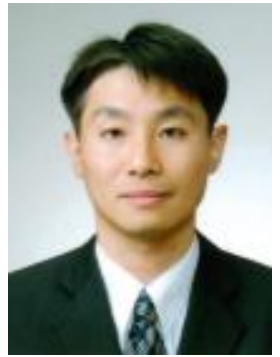

Shingo Yamaguchi is currently a (Full) Professor in the Graduate School of Sciences and Technology for Innovation, Yamaguchi University, Japan. He received the B.E., M.E. and D.E. degrees from Yamaguchi University, Japan, in 1992, 1994 and 2002, respectively. He was an Assistant Professor in the Faculty of Engineering, Yamaguchi University, from 1997 to 2007. He was also a Visiting Scholar in the Department of Computer Science at University of Illinois at Chicago, United States, in 2007. He was an Associate Professor in the Graduate School of Sciences and Technology for Innovation, Yamaguchi University, from 2007 to 2017. His research interest is in the area of theoretical computer science and software engineering, including their application to business process management, IoT, bigdata analysis, AI and cyber security. He is also a Senior Member of IEEE, Board of Governors of IEEE Consumer Electronics Society, Young Professionals Chair of IEEE Consumer Electronics Society, and a member of International Coordination Committee of ITC-CSCC. In academic societies other than IEEE and IEICE, he plays many important roles, which is an Editorial Board Member of International Journal of Internet of Things and Cyber-Assurance. 